\title{
Passenger Stability Within Moving Railway Vehicles: Limits on Maximum Longitudinal Acceleration
}

\author{
J. P. Powell ${ }^{1} \cdot$ R. Palacín ${ }^{1}$
}

Received: 9 March 2015/Revised: 17 May 2015/Accepted: 26 May 2015/Published online: 14 August 2015

(C) The Author(s) 2015. This article is published with open access at Springerlink.com

\begin{abstract}
Increasing the acceleration and deceleration of trains within a railway network can improve the performance of the system. However, the risk of passengers losing their balance and falling is also increased. The purpose of this paper is therefore to examine the effect of longitudinal vehicle accelerations on passenger safety and comfort. The literature review brings together two separate disciplinary areas, considering the effects of acceleration on balance from a physiological/kinesiological perspective, as well as looking at the results of previous empirical studies on the levels of acceleration that railway passengers will tolerate. The paper also describes an experiment carried out on the Tyne and Wear Metro, which gathered data on typical acceleration levels to compare against the findings of the literature review. It was found that both the magnitude of the accelerations and their rate of change (jerk) are important. The results also suggest that there may be scope to improve the trade-off between journey times, energy consumption and passenger comfort by fine control of the acceleration/jerk profile. This is particularly relevant to urban rail systems, as they typically feature relatively high acceleration and deceleration. However, the findings for passenger comfort are equally applicable to conventional regional and intercity services.
\end{abstract}

Keywords Passenger safety - Passenger comfort . Longitudinal acceleration · Braking · Jerk

J. P. Powell

j.powell2@newcastle.ac.uk

1 NewRail - Centre for Railway Research, Newcastle University, Stephenson Building, Claremont Road, Newcastle upon Tyne NE1 7RU, UK

Editor: Baoming Han

\section{Introduction}

An increase in the level of acceleration and deceleration achieved by a train allows a reduction of journey time (and a potential increase in railway system capacity), or alternatively a reduction in energy use for a given journey time. However, higher levels of longitudinal acceleration can compromise passenger comfort, and ultimately safety too if they are sufficient to cause passengers to lose their balance.

This has been highlighted as a significant cause of injury for bus passengers [10], with research suggesting that 'accelerations that are commonly encountered in practice appear to be impossible to endure without support [such as handgrips]' [7]. Although bus accelerations are typically higher than trains, passengers in a railway vehicle are more likely to be standing unsupported, or moving around within the vehicle. In Great Britain, the Rail Safety and Standards Board estimate that around $15 \%$ of on-board harm in the railway network in the last 10 years (measured by fatalities and weighted injuries) can be attributed to 'injuries attributable to sudden movements of the train due to lurching or braking' [21].

The purpose of this paper is therefore to examine the effects on railway passengers of the longitudinal accelerations found in regular operation, and the relationship to comfort and safety.

\section{Methodology and Paper Structure}

The outline methodology of this paper consists of two parts. The literature review in Sect. 3 first examines the biological theory unpinning balance, and then reconciles this with the results of previous empirical studies into the limits of acceleration that passengers will tolerate. 
Section 4 describes an experiment undertaken on the Tyne and Wear Metro to measure actual railway vehicle accelerations during regular operation, which are then compared against the findings from the literature, and some conclusions drawn in Sect. 5.

\section{Passenger Balance and Stability}

\subsection{Physiology/Kinesiology}

Balance in humans is an unconscious proprioceptive reaction, coordinated by the brain stem, supported by the cerebellum, visual cortex and basal ganglia. Information is obtained from the somatosensory system in the feet, the vestibular system in the inner ear and visual stimuli from the eyes [4]. The somatosensory system detects changes in pressure on the sole of the feet. When there is an imbalance between one foot and the other, it stimulates the muscles in the leg to contract so that the leg stiffens to oppose the increased pressure. The vestibular system consists of the semicircular canals and the otolith organs, and movement of fluid within each of these is detected by cells that stimulate the central nervous system. The semicircular canals provide a static response (effectively measuring position), and so help to stimulate corrective or predictive body movements such a stepping. The otolith organs provide a dynamic response and so control reflex reactions, such as flexing the body to change position. Finally, visual stimuli from the eyes provide an extra frame of reference to help determine position more accurately.

Following the above, three different strategies can be identified for retaining balance under the influence of an external acceleration. Where the acceleration is small, contracting the leg muscles and bending the ankle is sufficient to react against the external acceleration and keep the body balanced; this is known as ankle strategy. If the acceleration magnitude is greater, the body must change position to prevent falling, also bending at the hip. This is known as hip strategy, and requires a longer time for the muscles to actuate. Finally, the applied acceleration may be large enough that one or more steps must be taken to avoid falling; this is the stepping strategy.

Unconscious control of these strategies is a negative feedback system, therefore both the magnitude of the external acceleration and its rate of change (jerk) are important [31]. This implies that both strength and sensing/ actuation times of the body's muscles and nervous system must be considered when investigating the case of passengers balancing within a moving vehicle.

Where the jerk is very high, passengers will not have sufficient time to react, and their behaviour can be approximated by a static rigid body. This will topple when the line of action of the resultant force (due to external accelerations acting on it) lies outside of the base of support. This force will act through the body's centre of gravity, and for an average human this is approximately located at $54 \%$ of their height, in line with the front of their knee/ankle joints in a normal standing posture [24, 30].

The minimum time for muscles to react against external forces is typically $0.12-0.13 \mathrm{~s}[2,17]$, and for the body to make larger movements to retain balance takes around $1 \mathrm{~s}$ [25]. These figures may be considered to approximate the cases of the ankle and hip strategies respectively.

For a lower jerk level, the maximum tolerable acceleration is greater as the muscles have more time to actuate and resist the force. Where the jerk is very low, the strength of the individual will be the only important human factor, as the acceleration is changing slowly enough for the body to fully react and change posture as required.

Within a given population, there will be significant variation in the ability of individual passengers to balance under the influence of a given level of acceleration and jerk, in accordance with their physiology [4]. This variation means that it is difficult to set universal acceleration/jerk limits for passenger safety. It also means that, depending on their individual reactions to maintain balance, different passengers will have different perceptions of how uncomfortable a particular level of acceleration/jerk is.

\subsection{Review of Previous Experimental Work}

Empirical research into the levels of longitudinal acceleration that passengers will tolerate can be broadly classified into subjective and objective studies. A review was carried out by Hoberock [13] that included a mixture of both types, partly based on the work of Gebhard [9].

Subjective studies typically use questionnaires and interviews with study participants in order to establish how comfortable different acceleration profiles are for different people. A study by British Railways [16] was carried out to determine the effects on passengers of quasi-static lateral accelerations due to track curvature. For standing passengers, $0.1 \mathrm{~g}$ was given the approximate limit that could be attained without discomfort, and around $0.12 \mathrm{~g}$ was defined as uncomfortable. Values for seated subjects were somewhat higher, and it was also noted that lower levels of jerk increased the aforementioned acceleration limits. These values were found to have reasonable agreement with previous research in Britain [27] and by South African Railways [22]. The paper also demonstrated that passenger comfort on curves was generally a more limiting case than safety against derailment, and this formed the basis for British track design standards [6]. 
Hoberock also reported on similar experiments carried out by Japanese National Railways to assess passenger comfort during braking [18, 19], which also considered the effects of jerk. A later study [28] also included an evaluation of whether a given level of deceleration was acceptable to passengers, in addition to the assessments of comfort. It was noted that that the comfort ratings and the acceptability of different decelerations did not always correlate.

This research has been developed further by the Railway Technical Research Institute (RTRI) in Japan. Hiroaki [11] used questionnaires to examine the effect of high jerk values on the acceptability of different levels of acceleration. Curves for the acceptability of different levels of acceleration/jerk were produced, and an example for a group made up of regular commuters is illustrated in Fig. 1. Other curves were presented for occasional regional/intercity travellers, and the acceptability of a given acceleration/jerk level varied significantly depending on the type of passenger and journey being undertaken.

Overall, the variability in the methods of the subjective studies highlighted in this section means that they can only provide a general indication of what can be defined as acceptable or unacceptable levels, especially given the sensitivity of results to individuals' opinions or interpretations. Nonetheless, these studies confirm that both jerk and acceleration influence passenger comfort and stability, and also that unsupported standing passengers facing the direction of the vehicle's acceleration have the lowest tolerance.

Objective studies seek a quantifiable measure of people's reactions to external accelerations, rather than relying on their perception and opinion, and two significant studies were detailed by Hoberock. Hirshfeld [12] reported on a series of experiments intended to determine the effects of longitudinal acceleration on the loss of balance of standing passengers, as part of a wider design programme for the standardised PCC streetcars in the USA. Participants in the

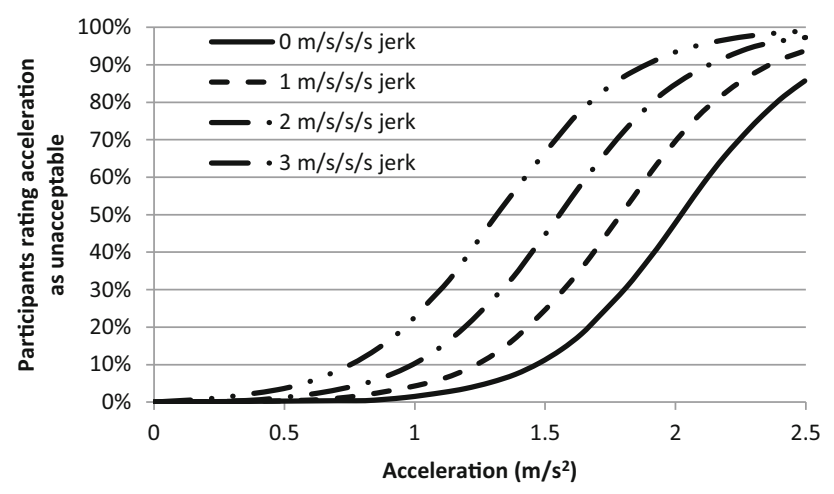

Fig. 1 Acceptability of acceleration/jerk levels [11] study stood on a platform that moved with variable acceleration profiles, and the average value of acceleration at which they either took a step or grabbed a handrail was measured. The study confirmed that different levels of jerk (for the same acceleration) influence the retention of balance, and that unsupported forward-facing passengers were least tolerant, losing their balance at an average of $0.13 \mathrm{~g}$. The combined average for all unsupported standees was $0.165 \mathrm{~g}$, increasing to $0.23 \mathrm{~g}$ with an overhead strap for support and $0.27 \mathrm{~g}$ with a vertical grab rail.

The experiments carried out by Browning [3] had similar objectives and methodology, although as part of a programme looking at the design of moving pedestrian walkways. The results were categorised in terms of the observed movement of the participants by an expert panel and presented in terms of acceleration against rise time (where jerk equals acceleration divided by rise time). Curves were produced for the approximate limits for each of the observed movement categories, and these are illustrated in Fig. 2.

Based on both the subjective and objective studies, Hoberock's principal conclusion was that it is difficult to set conclusive limits on acceleration and jerk, as passenger's reactions strongly depend on the individual concerned. A range was nonetheless suggested for maximum permissible accelerations of $0.11-0.15 \mathrm{~g}$ as an outline guide, with jerk limited to $0.30 \mathrm{~g} / \mathrm{s}$.

A more recent review [8] proposed 'large movement' in Browning's results to be approximately equivalent to Hirshfeld's case of passengers either stepping or requiring external support. The review also included acceleration values at which seated passengers start to be dislodged from their seats, based on the results of Abernethy et al. [1]. A limit for transverse (forward- or backward-facing) seats was given as $2.45 \mathrm{~m} / \mathrm{s}^{2}$, well above the guidelines for standing passengers, but a lower limit of $1.4 \mathrm{~m} / \mathrm{s}^{2}$ was given for longitudinal (side-facing) seats.

RTRI have also carried out further experimental studies that combine subjective and objective approaches [15], investigating the jerk limits required for high deceleration levels to be acceptable to passengers. The two graphs in Fig. 3 illustrate the data points and fitted curves for acceptability (left) and the ability of passengers to retain their balance (right) with four jerk levels.

A different type of experiment was carried out by Kamper et al. [14], in which the postural stability of a small group of wheelchair users with tetraplegia or paraplegia was examined under the influence of quasi-static accelerations typically found in road vehicles. $95 \%$ of the participants were able to retain balance within the wheelchair at an acceleration of $0.126 \mathrm{~g}$, and the average at which balance was lost was around $0.22 \mathrm{~g}$. Balance retention was improved with a lower level of jerk. 
Fig. 2 Browning's results, as summarised by Dorn [8]
Fig. 3 Subjective and objective results for forward-facing standing passengers [15]
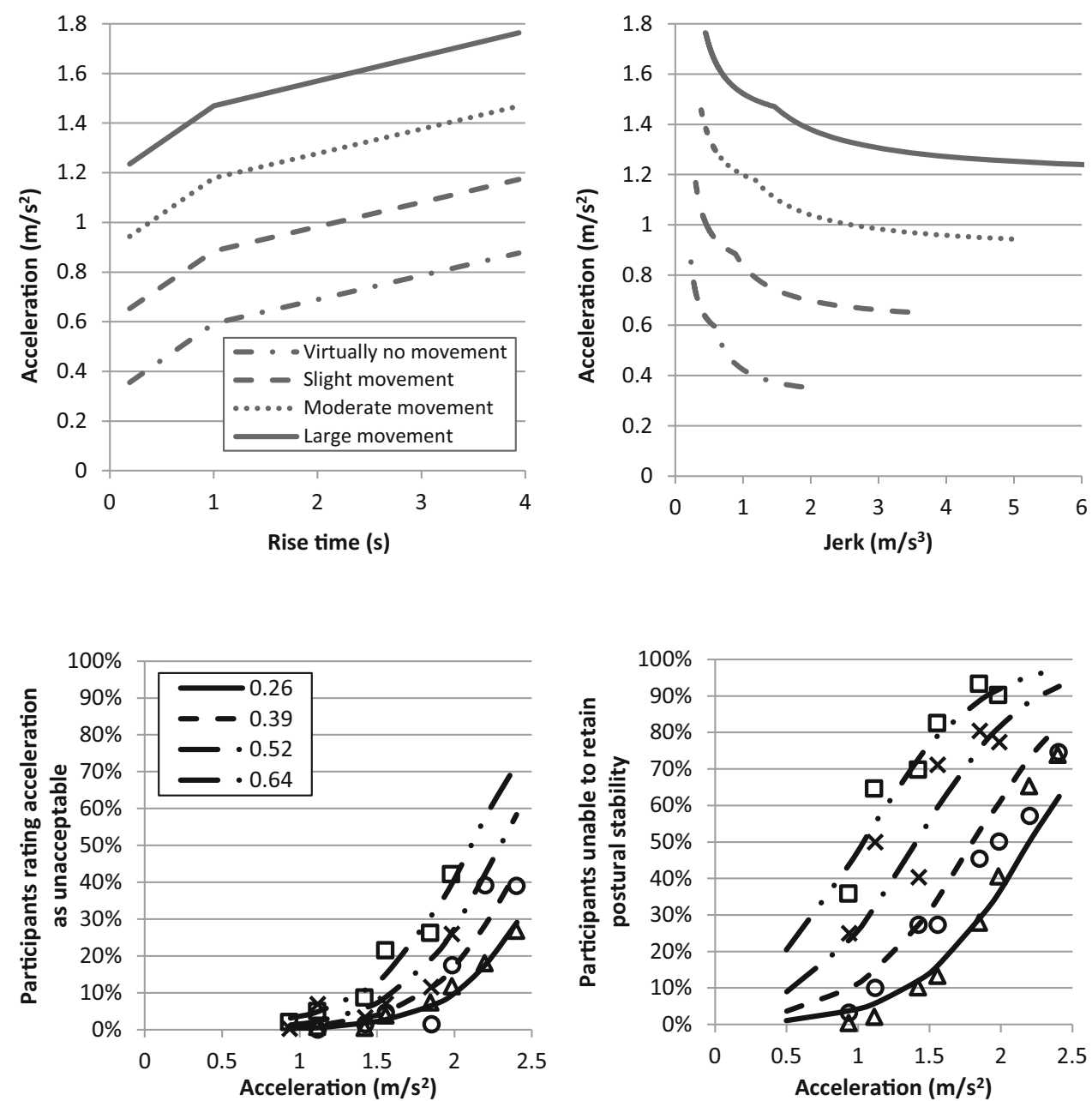

Finally, Sari [23] considered the likelihood of passengers walking within railway vehicles falling under the influence of low frequency $(0.5-2 \mathrm{~Hz})$ lateral oscillations. Although not directly applicable to this paper, as the accelerations are transient and the passengers are already in motion, it nonetheless provides a useful point of reference for comparison. A range of accelerations between 0.1 and $2.0 \mathrm{~m} / \mathrm{s}^{2}$ were tested across the range of frequencies. As may be expected, balance was generally lost at a lower acceleration level than the results for standing or seated passengers highlighted in this paper. Likewise, it was reported that results also varied with frequency of the oscillations, effectively the rate of change of these accelerations.

\subsection{Current Practice}

Although guideline figures for the safe limits of longitudinal accelerations in railway vehicles are used when specifying rolling stock, the source of the values is often unclear and can vary significantly [5, 20, 29]. Table 1 provides some examples from main line and light rail vehicles in Great Britain, from data provided by vehicle manufacturers/operators.

Note that some values given are estimates and should be taken as representative rather than exact. The values are the absolute maxima, and the average values achieved during braking in regular service are typically rather lower-this is illustrated further in Sect. 4 for the Tyne and Wear Metro. The operators also noted that emergency track brakes (indicated by an asterisk * in Table 1) are used as a last resort, as experience has shown their use carries a high risk of passenger injury.

\subsection{Findings}

Passenger safety becomes an issue when the acceleration/jerk levels require passengers to take one or more steps to retain balance (the stepping strategy), as this introduces the risk of falling. Passenger comfort is a more subjective measure, but may be considered quantitatively as how 
Table 1 Example maximum accelerations for railway vehicles in Great Britain

\begin{tabular}{llll}
\hline Vehicle & \multicolumn{3}{l}{ Maximum acceleration $\left(\mathrm{m} / \mathrm{s}^{2}\right)$} \\
\cline { 2 - 4 } & Traction & Service brakes & Emergency brakes \\
\hline Class 390 Pendolino (intercity EMU) & 0.37 & 0.88 & 1.18 \\
Class 156 Super Sprinter (regional DMU) & 0.75 & $0.7-0.8$ & $0.7-0.8$ \\
Class 323 (suburban EMU) & 0.99 & 0.88 & 1.18 \\
London Underground 1992 tube stock & 1.3 & 1.15 & 1.4 \\
Tyne and Wear Metrocar & 1.0 & 1.15 & $2.1\left(^{*}\right)$ \\
Manchester tram (Ansaldo T-68) & 1.3 & 1.3 & $2.6(*)$ \\
Sheffield Supertram (Siemens-Düwag) & 1.3 & 1.5 & $3.0(*)$ \\
Croydon tram (Bombardier FLEXITY) & 1.2 & 1.3 & $2.73(*)$ \\
Nottingham tram (Bombardier) & 1.2 & 1.4 & $2.5\left(^{*}\right)$ \\
\hline
\end{tabular}

close a particular individual is to their own limit of balance. This correlation is not exact however.

The RTRI studies [11, 15] provide an overview of the acceptability of acceleration/jerk for a population, while the results from Browning [3] provide some insight into the effects on individuals. There is a distinct change in the response at a rise time of around $1 \mathrm{~s}$, and it is proposed that this is due to the unconscious change from ankle strategy to hip strategy. For low values of jerk, or high values that correspond to a rise time of less than $0.12 \mathrm{~s}$, the acceleration value becomes independent of the jerk. It is instead related only to the strength of individual passengers for low values of jerk, or their posture and location of their centre of gravity for high values.

There is considerable variation between the perceptions and stability of different individuals however. This can be observed in the scatter in the results of Fig. 3, and more generally by the differences in the findings of the studies reviewed. It is therefore not possible to set precise passenger limits for longitudinal acceleration for passenger safety. Nonetheless, it can be concluded that previously suggested guidelines of 1.1 to $1.5 \mathrm{~m} / \mathrm{s}^{2}$ are reasonablethis is reflected in the current railway practice illustrated by Table 1 . The values also suggest that passengers are likely to be more tolerant of discomfort on short metrotype service by comparison with intercity/regional services.

\section{Measurement of Accelerations in Service}

\subsection{Introduction to Experimental Work}

An experiment was carried out on the Tyne and Wear Metro to measure the accelerations actually experienced by passengers in service, with the aim of comparing the findings of Sect. 3 against a mixture of subjective (qualitative) and objective (quantitative) data.
The Tyne and Wear Metrocars are towards the upper end of the range of accelerations given in Table 1. The Metro infrastructure consists of old railway alignments converted to Metro use, a new tunnel through Newcastle upon Tyne city centre (built specifically for the Metro), and sections of track shared with current heavy rail services. It therefore also features a range of curvature and gradient values typical of railway systems, including the highest values likely to be found on railway infrastructure. Although on-street tram systems can feature more extreme accelerations, passenger behaviour in trams is likely to be more similar to buses than railway vehicles. The Metro therefore provides a good case study for the purposes of this paper.

\subsection{Experimental Methods}

An afternoon peak time diagram was chosen for acceleration measurements, running empty from South Gosforth depot to Regent Centre, then in passenger service from Regent Centre to Pelaw and Pelaw to Monkseaton, before returning empty back to the depot.

The equipment consisted of three triaxial accelerometers with a range of $\pm 18 \mathrm{~g}$ and a multi-channel data acquisition system with a sample rate of $128 \mathrm{~Hz}$. This was set up within the depot in the B carriage of Metrocar 4007, on the double seat adjacent to the innermost door set (door 5). One accelerometer was glued to the overhead grab rail, one glued to the seat back, and one placed on a metal plate on the seat, sited so that passenger interference would be minimal. These are illustrated in Fig. 4. The accelerometers were calibrated by rotating through $\pm 90^{\circ}$ before final placement, effectively a $2 \mathrm{~g}$ inversion. The track in the depot where the equipment was installed was close to straight and level, minimising any offset in the readings (due to track geometry) during calibration.

During the test, a log was kept of arrival and departure times at each station, any other significant events (such as signal checks) and approximate passenger loadings. Notes 
Fig. 4 Experimental apparatus in situ on Metrocar 4007
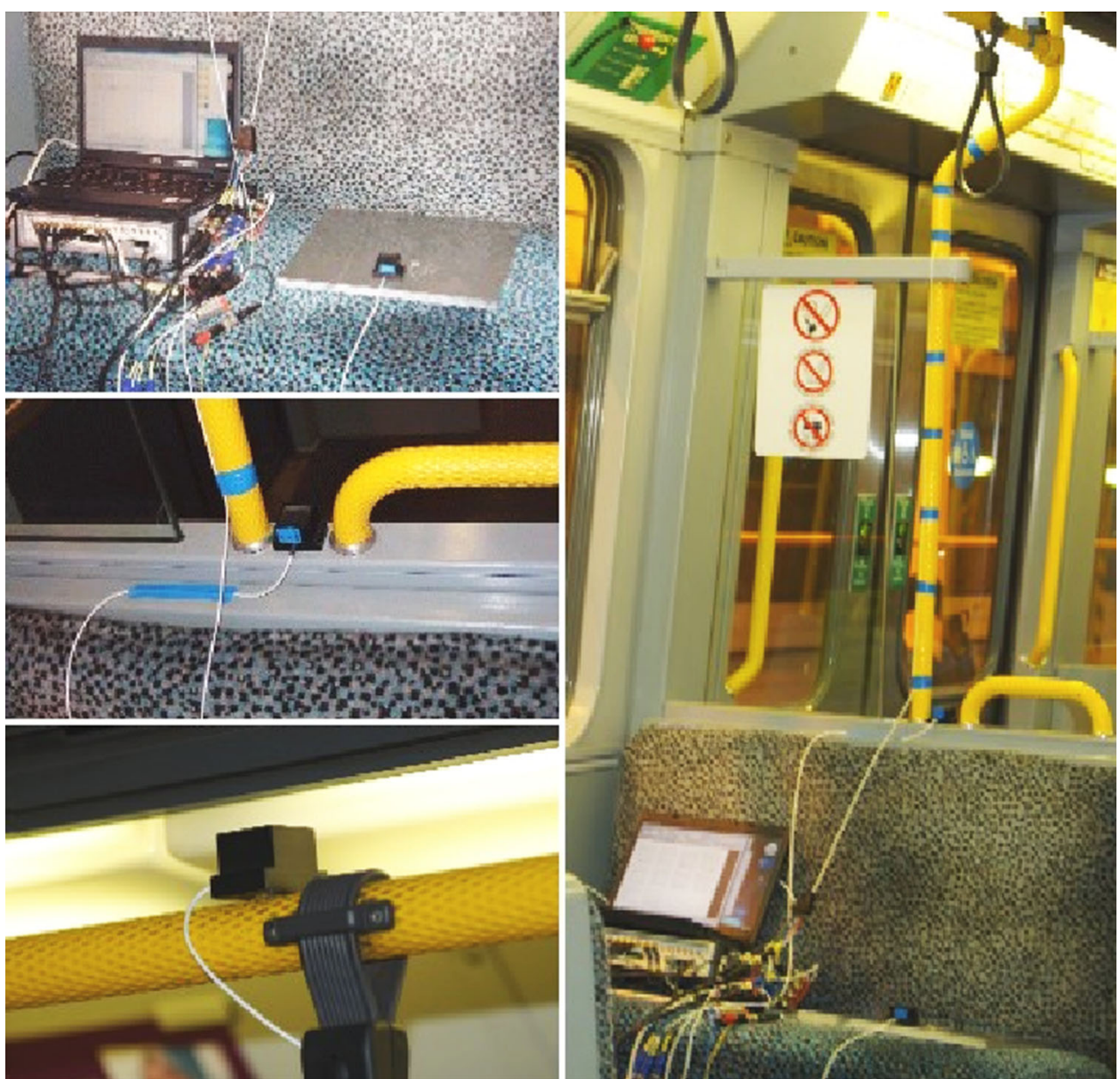

were also taken throughout the journey to qualitatively describe the comfort level at different locations.

\subsection{Results}

Figure 5 illustrates an example set of results for the accelerations measured in the longitudinal, lateral and vertical directions during the tests. The profile illustrated here includes section of running on old main line railway alignments, the tunnel designed specifically for Metro trains and track shared with heavy rail services.

Given that passengers may stand facing in any direction within the vehicle, the resultant of the lateral and longitudinal acceleration was calculated for each measured point and filtered to remove high frequency vibration in order to give the maximum quasi-static acceleration in the horizontal plane. For a static body, a vertical acceleration changes the effective weight (but not mass) of the body, changing the point at which it will topple. This mechanism was assumed to also apply to the cases of ankle and hip strategy, and the resultant horizontal plane acceleration was modified accordingly. The corresponding jerk for each measured point was then obtained by dividing the change in quasi-static acceleration between adjacent points by the sample time.

The wide variation in assessments of passenger comfort between different studies has already been noted. Therefore, the acceleration/jerk pairs from the measured data were compared against the proposed curves in both Figs. 2 and 3. There were several locations where the measured acceleration/jerk pairs were highlighted as problematic by these methods, and these matched up well with the subjective observations on comfort recorded during the test. These locations were therefore analysed to look for patterns that might suggest how to improve passenger comfort.

\subsection{Discussion}

The majority of the cases where acceleration/jerk levels were outside the proposed limits were found to be when the train was stopping at a station, as it came to a standstill. By definition, the jerk approaches infinity at the moment speed equals zero when stopping, and therefore a lower acceleration magnitude is necessary. It is common driving practice to reduce the braking effort demanded as the train comes to 


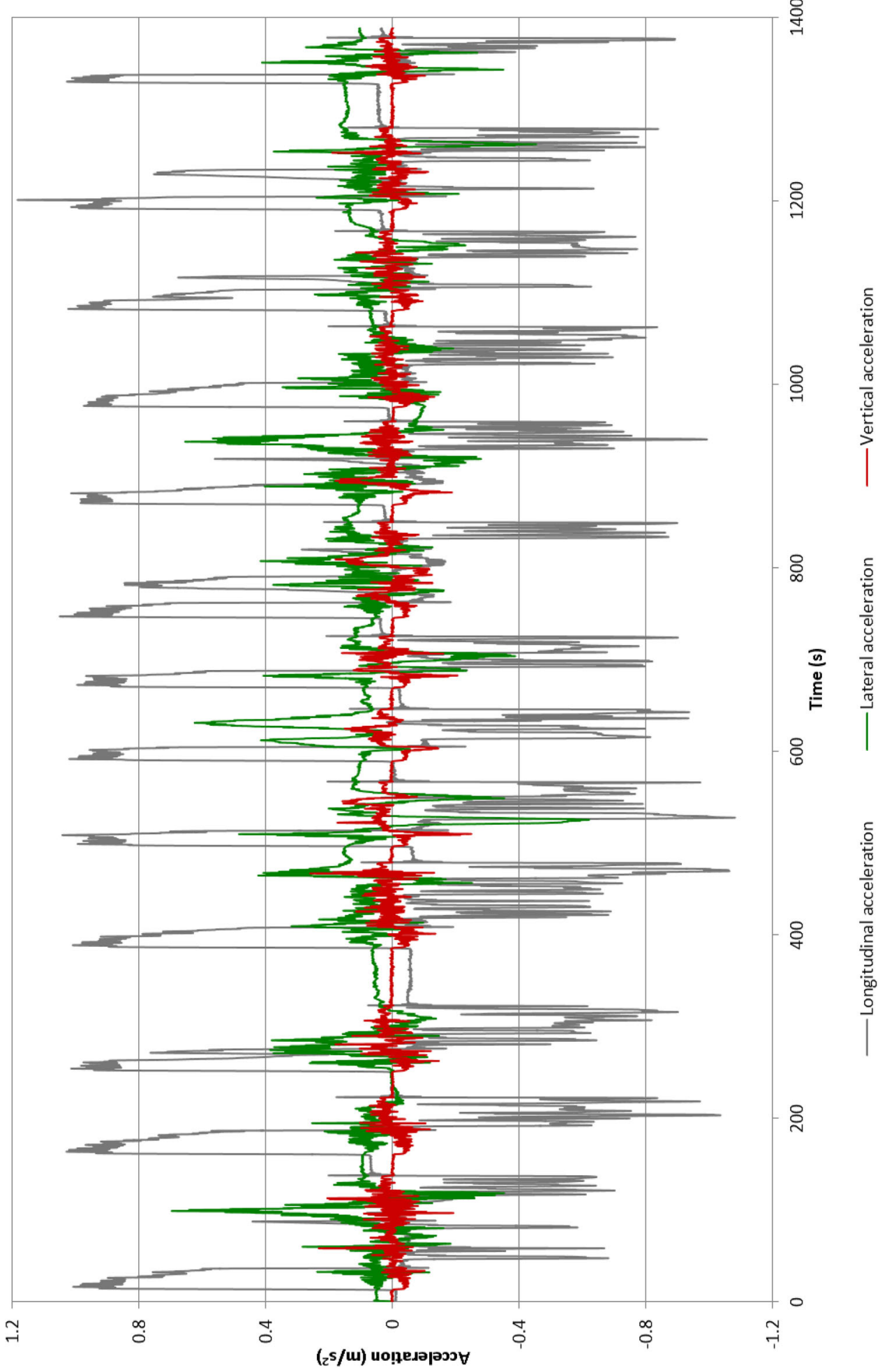

 
a standstill to address this. Sone and Ashiya [26] provide an example of how pure electric braking (rheostatic or regenerative, using three phase $\mathrm{AC}$ traction motors) can achieve superior passenger comfort in this respect.

The worst case measured was when traction power was cut off at a relatively low speed while accelerating, in order to meet a speed restriction. The Tyne and Wear Metrocars have camshaft resistance control of DC traction motors, and although the first couple of camshaft steps limit the jerk when power is first applied, power is cut off abruptly by contactors when the combined power/brake controller is returned to neutral. As in the previous case, this results in a large jerk. However, the camshaft control also means that driver has less control over the level of tractive effort by comparison with braking, and for relatively low speeds the train's acceleration will be close to its maximum level. This situation occurs far less often than station stops, but passengers are less likely to be able to predict and anticipate it, and the subjective observations on the Metro suggest it is also the most likely reason for passengers making large movements to correct their balance.

There were also a few cases where the train changed immediately from accelerating to braking, or where the driver was moving the controller frequently between discrete brake notches while decelerating. This can be observed in Fig. 5, where the negative longitudinal accelerations during braking show significantly more variation than the positive longitudinal accelerations. The locations at the cases highlighted generally also coincided with specific infrastructure features that influence the acceleration and jerk levels, such as sharp curves and their associated transitions, which increased the lateral acceleration and jerk. Automatic Train Operation (ATO) can mitigate this variation and provides a more consistent control of braking effort, improving passenger comfort.

Overall, resultant quasi-static accelerations were routinely observed approaching $1.4 \mathrm{~m} / \mathrm{s}^{2}$. The majority of these cases were found to be acceptable by both the qualitative observations during the test and the quantitative data collected-the locations identified as problematic were not necessarily the locations with a high acceleration level, but in all cases did involve a high value of jerk.

\section{Conclusions}

Passenger tolerance to longitudinal accelerations varies significantly between different individuals, according to their physiology and psychology. The acceptability of a given level of acceleration depends strongly on the rate of change of the acceleration (jerk).

Acceleration and jerk limits are typically given as single figures in rolling stock specifications. However, this paper suggests that there may be scope to improve the trade-off between journey times, energy consumption and passenger comfort by fine control of the acceleration/jerk profile. ATO and electric braking (using three phase AC traction motors) are likely to be a prerequisite for the level of control required however.

This research is particularly applicable to urban rail systems, as they typically operate vehicles at higher acceleration and deceleration levels than conventional regional or intercity passenger trains. However, passengers on regional or intercity services are likely to expect a higher level of comfort than urban rail passengers, and control of the jerk then becomes important in this respect.

Acknowledgments The authors would like to thank Dr Kazuma Nakai of RTRI for his assistance with the Japanese research and helpful suggestions for the paper, and Nexus for the opportunity to carry out experimental work on the Tyne and Wear Metro (the work described here was carried out when the Metro was managed as an integrated system, prior to train operations being split from infrastructure and contracted separately).

Open Access This article is distributed under the terms of the Creative Commons Attribution 4.0 International License (http://crea tivecommons.org/licenses/by/4.0/), which permits unrestricted use, distribution, and reproduction in any medium, provided you give appropriate credit to the original author(s) and the source, provide a link to the Creative Commons license, and indicate if changes were made.

\section{References}

1. Abernethy CN, Jacobs HH, Plank GR, Stoklosa JH, Sussman ED (1980) Maximum deceleration and jerk levels that allow retention of unrestrained, seated transit passengers. Transp Res Rec $774: 45-51$

2. Allum JHJ (1983) Organization of stabilizing reflex responses in tibialis anterior muscles following ankle flexion perturbations of standing man. Brain Res 264(2):297-301

3. Browning AC (1972) The tolerance of the general public to horizontally accelerating floors, with special reference to pedestrian conveyors. Technical Report TR71105, Royal Aircraft Establishment, Ministry of Defense

4. Carpenter RHS (2003) Neurophysiology, 4th edn. Arnold, London

5. Cole C (2006) Longitudinal train dynamics. In: Iwnicki S (ed) Handbook of railway vehicle dynamics. CRC/Taylor \& Francis, Boca Raton, pp 239-277

6. Cope GH (1993) British railway track: design, construction and maintenance, 6th edn. Permanent Way Institution, Barnsley

7. De Graaf B, Van Weperen W (1997) The retention of balance: an exploratory study into the limits of acceleration the human body can withstand without losing equilibrium. Hum Factors: J Hum Factors Ergon Soc 39(1):111-118

8. Dorn MR (1998) 'Jerk, acceleration and the safety of passengers' Technology for Business Needs. Birmingham, 24th-26th November

9. Gebhard JW (1970) Acceleration and comfort in public ground transportation. Transportation Programs Report 002, The Johns Hopkins University

10. Halpern P, Siebzehner MI, Aladgem D, Sorkine P, Bechar R (2005) Non-collision injuries in public buses: a national survey of a neglected problem. Emerg Med J 22(2):108-110 
11. Hiroaki S (1995) Ability to withstand sudden braking. Railw Res Rev 52(5):18-21

12. Hirshfeld CF (1932) Disturbing effects of horizontal acceleration. Bulletin No. 3, Electric Railway President's Conference Committee

13. Hoberock LL (1976) A survey of longitudinal acceleration comfort studies in ground transportation vehicles. Research Report 40, Council for Advanced Transportation Studies

14. Kamper D, Parnianpour M, Barin K, Adams T, Linden M, Hemami H (1999) Postural stability of wheelchair users exposed to sustained, external perturbations. J Rehabil Res Dev 36(2): $121-132$

15. Koji O, Hiroharu E, Hiroaki S, Hiroshi S (2007) Ride evaluation during high deceleration braking. Railw Res Rev 64(7):26-29

16. Loach JC, Maycock MG (1952) Recent developments in railway curve design. ICE Proc: Eng Div 1(5):503-541

17. Maki BE, Fernie GR (1988) A system identification approach to balance testing. In: Pompeiano O, Allum JHJ (eds) Progress in brain research. Elsevier, Oxford, pp 297-306

18. Matsudaira T (1960) Dynamics of high speed rolling stock, Railway Technical Research Institute, Quarterly Reports, (Special Issue), pp 57-65

19. Matsui S (1962) Comfort limits of retardation and its changing rate for train passengers. Jpn Railw Eng 3(11):25-27

20. Profillidis VA (2006) Railway management and engineering, 3rd edn. Ashgate, Aldershot
21. RSSB (2014) Annual Safety Performance Report 2013/14. Rail Safety and Standards Board

22. SAR (1948) Superelevations and maximum permissible speeds on curves. Research Circular No. 25.027, South African Railways

23. Sari MH (2012) Postural stability when walking and exposed to lateral oscillations. Doctoral thesis, University of Southampton

24. Shumway-Cook A, Woollacott MH (2001) Motor control: theory and practical applications. Lippincott Williams \& Wilkins, Philadelphia

25. Simoneau M, Corbeil P (2005) The effect of time to peak ankle torque on balance stability boundary: experimental validation of a biomechanical model. Exp Brain Res 165(2):217-228

26. Sone S, Ashiya M (1998) An innovative traction system from the viewpoint of braking, International Conference on Developments in Mass Transit Systems. London, 20th-23rd April

27. Thompson JT (1939) Railway track-work for high speeds. J ICE 10(3):405-407

28. Urabe S, Nomura Y (1964) Evaluations of train riding comfort under various decelerations, Railway Technical Research Institute, Quarterly Reports 5(2):28-34

29. Vuchic VR (2007) Urban transit systems and technology. John Wiley \& Sons, Hoboken

30. Watkins J (1983) An introduction to mechanics of human movement. Boston, [Mass.]; Lancaster: MTP Press

31. Zigmond MJ (1999) Fundamental neuroscience. Academic, San Diego 